\title{
Surface Modifications of Support Partitions for Stabilizing Biomimetic Membrane Arrays
} \author{
Maike Benter ${ }^{4}$ and Claus Hélix-Nielsen ${ }^{1,5 *}$ \\ ${ }^{1}$ Aquaporin A/S, Ole Maaløes Vej 3, DK-2200 Copenhagen, Denmark \\ 2DTU Nanotech, Technical University of Denmark, DK-2800 Lyngby, Denmark \\ ${ }^{3}$ NanoScience Center, Copenhagen University, Universitetsparken 5, DK-2100 Copenhagen Ø, Denmark \\ ${ }^{4}$ Nanon A/S, Priorparken 878, 2605 Brøndby, Denmark \\ ${ }^{5}$ DTU Physics, Technical University of Denmark, DK-2800 Lyngby, Denmark \\ \#These authors have contributed equally to the work presented
}

Mark Perry ${ }^{1 \#}$, Jesper S. Hansen ${ }^{1,2 \#}$, Karin Stibius ${ }^{1,5 \#}$, Thomas Vissing ${ }^{1}$, Kamila Pszon-Bartosz $^{1,2}$, Christian Rein $^{1,3}$, Bahram Eshtehardi $^{4}$

\begin{abstract}
Black lipid membrane (BLM) formation across apertures in an ethylene tetra-fluoroethylene (ETFE) partition separating two aqueous compartments is an established technique for the creation of biomimetic membranes. Recently multi-aperture BLM arrays have attracted interest and in order to increase BLM array stability we studied the effect of covalently modifying the partition substrate using surface plasma polymerization with hydrophobic $n$-hexene, 1-decene and hexamethyldisiloxane (HMDSO) as modification groups. Average lifetimes across singlesided HMDSO modified partitions or using 1-decene modified partitions were similar and significantly lower than for arrays formed using untreated ETFE partitions. For single side $n$-hexene modification average membrane array lifetimes were not significantly changed compared to untreated ETFE. Double-sided $n$-hexene modification greatly improved average membrane array lifetimes compared to membrane arrays formed across untreated ETFE partitions. $n$-hexene modifications resulted in BLM membrane arrays which over time developed significantly lower conductance $\left(G_{m}\right)$ and higher capacitance $\left(C_{m}\right)$ values compared to the other membranes with the strongest effect for double sided modification. $n$-hexene modification is evident as a change in surface energy whereas the surface roughness does not change significantly. The concomitant low $G_{m}$ and high $C_{m}$ values for BLM arrays formed using double-sided $n$-hexene modification enable transmembrane ionic current recordings with a high signal-to-noise $(s / n)$ ratio. We demonstratesd this by reconstituting gA and $\alpha$-hemolysin $(\alpha-H L)$ into BLM arrays. The improvement in membrane array lifetime and $s / n$ ratio demonstrates that surface plasma polymerization of the supporting partition can be used to increase the stability of biomimetic membrane arrays.
\end{abstract}

Abbreviations: $\alpha$-Hemolysin: $\alpha-H L ;$ AFM: Atomic Force Microscopy; BLM: Black Lipid Membrane; DPHPC: 1,2-Diphytanoylsn-Glycero-3-Phosphocholine; ETFE: Ethylene Tetrafluoroethylene; HMDSO: Hexamethyldisiloxane

\section{Introduction}

Biomimetic membranes with embedded proteins are increasingly attracting attention because of their properties as model cell membranes, which can be employed in a variety of fundamental biological studies and medical and technological devices $[15,16]$. The latter have both scientific and practical interest because of their potential applications in the field of biosensors, drug screening, tissue engineering, and medical implants [12]. Amongst the general challenges in biomimetic membrane design is to scale up membrane effective areas, to create stable (addressable) membrane arrays with long (>days) lifetimes [6]. Specifically, it is important to ensure that the membranes have low ionic permeability as evidenced by a low value for the electrical conductance $\left(\mathrm{G}_{\mathrm{m}}\right)$ in particular for sensors based on ion channels embedded in biomimetic membrane arrays. Also, a high value for the membrane capacitance $\left(\mathrm{C}_{\mathrm{m}}\right)$ is desirable in sensors based on detecting changes in membrane impedance. Generally, the formation, stability, and electrical properties of large stable membranes are critically dependent on the coupling between the membrane and its supporting scaffold $[8,22,23]$. In the development of biomimetic membranes it therefore becomes important to ensure good coupling/ adhesion at the interface between the biomimetic membrane and its surrounding scaffold.

Black lipid membrane (BLM) formation across apertures in a hydrophobic scaffold or partition, typically ethylene tetrafluoroethylene (ETFE), separating two aqueous compartments is an established technique for the creation of biomimetic membranes [19]. BLMs can be established principally by two basic techniques; the Mueller-Rudin painting technique [16] and the Montal-Mueller folding technique (Montal and Mueller 1972), respectively. Painted BLMs are formed by depositing a solvent (hydrocarbon) containing lipid solution across a partition aperture of up to $1 \mathrm{~mm}$ in diameter and then allow the solution to thin out (as evidenced by an increase in $\mathrm{C}_{\mathrm{m}}$ ) so that a lipid bilayer is formed surrounded by a solvent containing torus. Folded BLMs are formed by spreading lipid monolayers at the air-water interface in each compartment and then raising the aqueous solution resulting in a BLM formed across the partition aperture with typical diameters of $50-100 \mu \mathrm{m}$.

Common for both the painting and folding technique for establishing BLMs is the requirement for a partition pretreatment step before stable lipid bilayers with low $\mathrm{G}_{\mathrm{m}}$ values - i.e. tight membranes - and high $\mathrm{C}_{\mathrm{m}}$ values - i.e. large effective bilayer areas - can be created across the partition aperture. The pretreatment typically consists of applying a hydrocarbon solvent to the partition, which is subsequently

*Corresponding author: Claus Hélix-Nielsen DTU Physics, Fysikvej 309, Building 309, Office 138 Technical University of DenmarkDK-2800 Lyngby, Tel: +456068 10 81; Fax: +45 459316 69; Email: Claus.Helix.Nielsen@fysik.dtu.dk

Received May 16, 2011; Accepted June 06, 2011; Published November 15, 2011

Citation: Perry M, Hansen JS, Stibius K, Vissing T, Pszon-Bartosz K, et al. (2011) Surface Modifications of Support Partitions for Stabilizing Biomimetic Membrane Arrays. J Membra Sci Technol S1:001. doi:10.4172/2155-9589.S1-001

Copyright: (c) 2011 Perry M, et al. This is an open-access article distributed under the terms of the Creative Commons Attribution License, which permits unrestricted use, distribution, and reproduction in any medium, provided the original author and source are credited. 
Citation: Perry M, Hansen JS, Stibius K, Vissing T, Pszon-Bartosz K, et al. (2011) Surface Modifications of Support Partitions for Stabilizing Biomimetic Membrane Arrays. J Membra Sci Technol S1:001. doi:10.4172/2155-9589.S1-001

allowed to dry onto it prior to filling the chamber compartments with the electrolyte solution [22]. The partition pretreatment step provides a proper interaction of the lipid molecules around the aperture edge which ensures stabilization of the lipid bilayer. A high degree of stabilization is directly reflected in a low value of $G_{m}$ and the pretreatment step creates essential conditions for establishing stable biomimetic membranes [19].

Recently, multi-aperture BLM arrays have attracted interest as a method to scale up the effective area of biomimetic membranes [8]. Functional biomimetic membranes formed across multiple apertures requires a high degree of stability and production reproducibility and both parameters are strongly influenced by the partition material properties. Previously we described the development of an automation technique for establishing BLMs in arrays and we showed that variations in the pretreatment of the ETFE partition was a major determining factor for the ability to reproducibly establish lipid bilayers [8]. The reproducibility could however be increased significantly by developing a controllable airbrush pretreatment technique. Although a high reproducibility was achieved with the automation technique for establishing lipid bilayers, the low leak currents of established bilayers could only be maintained for approximately 100 min, meaning that membrane lifetimes were not increased concomitantly.

In this study we addressed the stability of painted BLMs as a function of changing the chemical composition of the surface of the partition substrate - mimicking the traditional prepainting. Plasma polymerization is a well-known technique for changing the chemical properties of the top 10-100 nm of a substrate surface while maintaining the bulk physio-chemical substrate properties [24]. Previous studies have shown that plasma polymerization can be used to modify the otherwise chemically inert surface of ETFE and be used to introduce covalently bound surface functionalities onto substrates [9-11,17]. We employed plasma polymerization (see Figure 1A) to add hydrophobic groups to the ETFE substrate material mimicking the traditional prepainting step and evaluated the effects of surface modification on lipid bilayer stability and reproducibility.

We investigated three different monomers for surface modification: hexamethyldisiloxane (HDMSO) 1 -decene and $n$-hexene (see Figure 1B-D). HDMSO was chosen because of its ability to form hydrophobic layers on polymeric films by plasma polymerization $[2,26]$, The olefins 1 -decene and $n$-hexene were chosen as a mimic to traditional prepainting using a low molecular weight hydrocarbon [8].

We demonstrate that by using double-sided plasma polymerization with $n$-hexene it is possible to obtain stable (i.e. long lived) biomimetic lipid membrane arrays with high $\mathrm{C}_{\mathrm{m}}$ values (large effective areas) and good adhesion as reflected by low $\mathrm{G}_{\mathrm{m}}$ values. We suggest that plasma polymerization may provide an efficient method to create substrates suitable for establishing reproducibly and long-lived bilayer lipid membranes. Moreover, the surface modification can be tailor made for specific membrane forming solutions.

\section{Experimental}

\section{Reagents and materials}

Tefzel ethylene tetrafluoroethylene (ETFE) LZ200 fluoropolymer for the fabrication of multi aperture partitions were from DuPont Fluoropolymers (Detroit, U.S.A). Sheets of regenerated cellulose (DSSRC70PP) were purchased from Alfa Laval (Nakskov, Denmark). Round glass cover slips $(30 \mathrm{~mm})$ were from VWR - Bie \& Berntsen (Herlev, Denmark). $n$-hexene from Fluka, purity $\geq 96 \%$, hexamethyldisiloxane
(HMDSO) from ABCR purity $98 \%$, and 1-decene from Aldrich, purity $\geq 94 \%$ were used for plasma polymerization and were purchased from Sigma-Aldrich Denmark (Brøndby, Denmark). Diphytanoylsn-Glycero-3-Phosphocholine was from Avanti Polar Lipids Inc. (Alabaster, U.S.A). Bacillus brevis gAA (gA) (Sigma), Staphylococcus aureus $\alpha$-Hemolysin (Sigma), 99\% ethanol, $n$-heptane and $n$-decane (Fluka) were purchased from Sigma-Aldrich Denmark (Brøndby, Denmark). All other chemicals were of analytical grade and purchased from commercial sources.

\section{Micro structuring and surface modification of ETFE partitions}

ETFE LZ200 film (50.8 $\mu \mathrm{m}$ thickness) was laser structured to produce partition aperture arrays as previously described [6]. Partitions produced were multi-aperture partitions rectangular with $8 \times 8$ arrays of apertures with $300 \mu \mathrm{m}$ aperture diameters and nominal centre-tocentre distances of $400 \mu \mathrm{m}$.

\section{Surface plasma polymerization and characterization}

Surfaces of ETFE were plasma polymerized with HMDSO, 1 -decene, and $n$-hexene in a custom built plasma chamber with a chamber volume of $30 \mathrm{~L}$ using argon (Ar) as activation and carrier gas for a sketch of the chamber set up see Figure 1A) and for a general reference please refer to (Yasuda 1985. ). The parameters for the plasma treatments are tabulated in the appendix. All surfaces of ETFE

A)

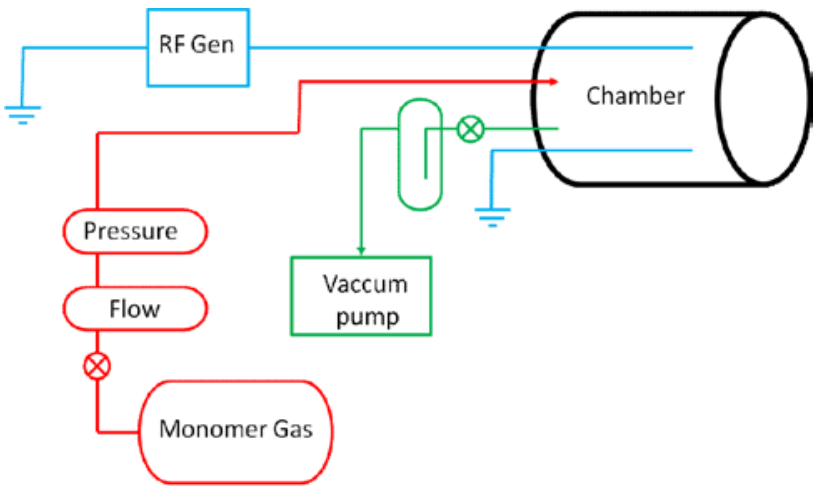

B)

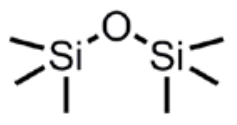

C)

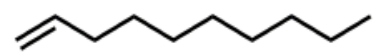

D)

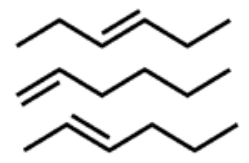

Figure 1: Plasma chamber sketch and monomer structures. A): Diagram of the plasma chamber. The chamber (black outline) is evacuated by a vaccum line (green) where a pump is connected via a trap to collect residual monomers. Electric fields are generated using a generator and antenna in the chamber (blue system). Monomers are supplied as a flow with $\mathrm{Ar}$ as carrier gas (red system). B): Hexamethyldisiloxane (HDMSO) monomer. C): 1-decene monomer. D): $n$-hexene monomers. 

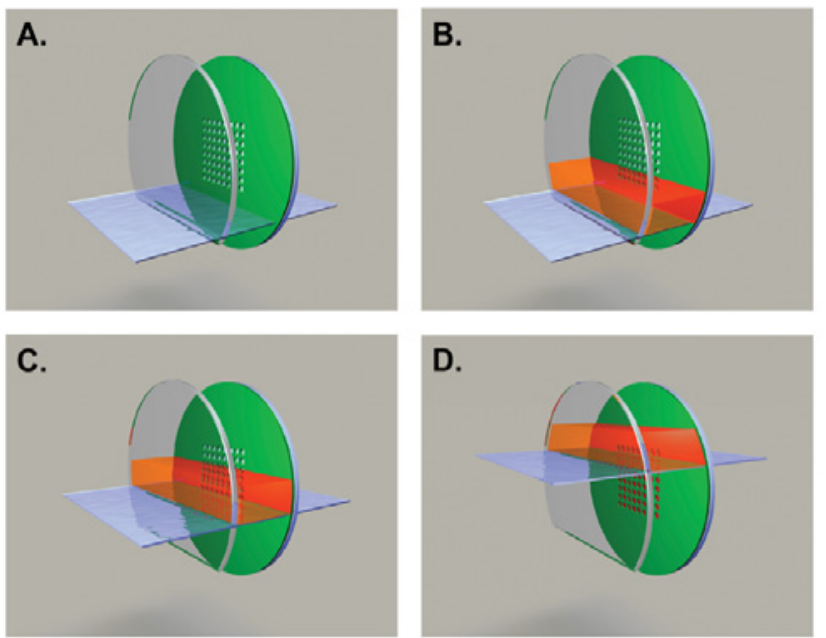

Figure 2: Principle for establishment of $B L M$ arrays. The aqueous electrolyte solution (is filled up to the cut glass cover slip (A) and the BFS (red) is applied from the top $(B)$. The aqueous electrolyte solution is then slowly applied to the cis chamber thereby raising the BFS across the $8 \times 8$ aperture partition (green) to form an array of lipid bilayers (C-D).

were cleaned with ethanol (EtOH) and MilliQ water prior to plasma treatment. The Softplasma ${ }^{\mathrm{TM}}$ technique for treating ETFE partitions can be divided into an activation step and polymerisation step. In the activation step, a $40 \mathrm{kHz}$ radio frequency (RF) or $50 \mathrm{~Hz}$ Argon plasma is created in the plasma chamber at a medium vacuum between 5 and $15 \mathrm{~Pa}$. Both the partial pressure and activation time are lower during the activation step as compared to the polymerisation step.

Upon surface activation, the monomer in its gaseous phase is pumped into the plasma chamber. For the surface treatment of our model ETFE partitions a polymerisation time of 7-12 minutes has been found to produce a monomer thickness that renders our samples resistant to our cleaning procedures. After polymerisation the plasma is broken by switching of the power and any non-reacted monomer is pumped out of the chamber. Finally the vacuum is broken by letting air into the plasma chamber. Throughout the procedure mass flow controllers are used to measure Argon and monomer flow. For singlesided treatments the samples were laid out on a glass surface in the chamber, whereas for the double-sided treatments the partitions were mounted in a small grooved aluminum block.

Following plasma polymerization treatment, the water contact angle of the samples was measured using DataPhysics OCA 15 (Filderstedt, Germany) and the samples were characterised using Fourier Transform Infrared/Attenuated Total Reflection (FTIR/ATR ) spectroscopy using Thermo Nicolet Nexus 470 Spectrometer (Thermo Scientific Denmark, Copenhagen, Denmark). The spectra were obtained by ATR on a Gemirror. The solvent stability of the plasma coatings were tested by analyzing water contact angles and FTIR spectral features before and after rinsing the surface modified samples in three successive rinsing cycles of $60: 40 \mathrm{v} / \mathrm{v}$ EtOH:water, $n$-heptane, and MilliQ water.

$n$-hexene modified ETFE partitions were examined using atomic force microscopy (AFM). For comparative measurements a 'half $n$-hexene treated surface was prepared by $n$-hexene treating an ETFE partition while protecting part of the surface by a glass slide during plasma modification. The sample was then glued with epoxy to a silicon wafer base. Measurements were made on samples in MilliQ water with an Asylum Research MFP-3D AFM instrument (Asylum Research,
Santa Barbara, CA, US) using a hexadecanethiol modified Au-coated Bio-Lever probe (Olympus Denmark, Ballerup, Denmark) with a nominal tip radius of curvature of $30 \mathrm{~nm}$. The probe was stored in 0.1 $\mathrm{MM}$ hexadecanethiol/EtOH for two weeks. Initial stress of the probe was released by allowing it to equalize for 1 hour before measurements. Adhesion energy value maps were calculated by integrating forcedistance curves (contact mode) for each pixel $(1.56 \mu \mathrm{m} \times 1.56 \mu \mathrm{m})$ using an algorithm provided by Asylum Research. The probe was calibrated on the silicon wafer base (Olympus Denmark, Ballerup, Denmark) with contact angle $\sim 40^{\circ}$, and a reference force volume (FV) map was made.

\section{Preparation of lipid solutions and formation of BLM arrays}

The bilayer forming solution (BFS) consisted of 1, 2-Diphytanoyl$s n$-Glycero-3-Phosphocholine (DPhPC) in $n$-decane $(50 \mathrm{mg} / \mathrm{ml})$. The lipid solution was prepared the day before, and stored at $-20^{\circ} \mathrm{C}$ until use. Planar lipid bilayers were established across the surface modified ETFE partition arrays by the lipid bilayer automation technique as previously described [8], see Figure 2. Briefly, the two-cell lipid bilayer chamber was assembled with a surface modified $8 \times 8$ multi aperture ETFE partition array and with a circular regenerated cellulose sheet (DSS-RC70PP, Alfa Laval, Nakskov, Denmark). This porous semisupported bilayer design prevents BFS flow through the apertures from the trans to the cis chamber upon establishment of lipid bilayers. The trans chamber was subsequently filled with $7.5 \mathrm{ml} 0.2 \mathrm{M} \mathrm{KCl}$ saline solution, whereas the cis chamber was added $0.5 \mathrm{ml}$ of the saline solution. The lipid bilayer solution $(100 \mu \mathrm{l})$ was then carefully added to the cis chamber using a Hamilton pipette. The level of the aqueous electrolyte solution in the cis chamber was then slowly raised by adding approximately $7 \mathrm{ml}$ of the saline solution using a plastic Pasteur pipette. In this way lipid bilayers were created across all of the 64 apertures of the ETFE partition arrays. The formation of lipid bilayers in the partition aperture arrays was recorded by voltage-clamp measurements of the membrane $\mathrm{C}_{\mathrm{m}}$ and $\mathrm{G}_{\mathrm{m}}$ values [18].

\section{Voltage-clamp data acquisition and processing}

The experimental setup consisted of a Model 2400 Patch Clamp Amplifier with a head stage containing $10 \mathrm{G} \Omega / 10 \mathrm{M} \Omega$ feedback resistors (A-M Systems, Inc., WA, USA) and a Thurlby Thandar Instruments model TG2000 20MHz DDS function generator (RS Components Ltd, Northants, UK). The $\mathrm{Ag} / \mathrm{AgCl}$ electrodes were placed in the trans and cis compartments chamber with the ground electrode positioned in the trans compartment. $\mathrm{G}_{\mathrm{m}}$ and $\mathrm{C}_{\mathrm{m}}$ were determined from responses to rectangular and triangular waveforms $\left(20 \mathrm{mV}_{\mathrm{pp}}\right.$ and $\left.50 \mathrm{~Hz}\right)$. The capacitance of the perforated ETFE partition in electrolyte only (i.e. no membranes) is $<100 \mathrm{pF}$. Data acquisition was done with a combined oscilloscope/analog-digital converter (ADC-212/50, Pico Technology, Cambridgeshire, UK) connected to a laptop computer. Sampling frequency was $50 \mathrm{~Hz}$. Off-line analysis was done using custom made software [18]. For the gAchannel activity measurements the peptide was added from ethanolic stock solution to the BFS to a final protein:lipid ratio of 1:107. Staphylococcus aureus $\alpha$-Hemolysin ( $\alpha-\mathrm{HL})$ was incorporated by adding $10 \mu \mathrm{l}$ of a $0.5 \mathrm{mg} / \mathrm{ml}$ PBS stock solution to the cis chamber. For the gAexperiments the aqueous solution was $1 \mathrm{M} \mathrm{HCl}$ and for the a-HL experiments the aqueous solution in the chamber consisted of phosphate buffered saline (PBS). The applied potential across the partition arrays was $+60 \mathrm{mV}$.

\section{Results and Discussion}

\section{Plasma funtionalization results in robust partition coatings}

All samples were characterized using FTIR/ATR and the results 
A)

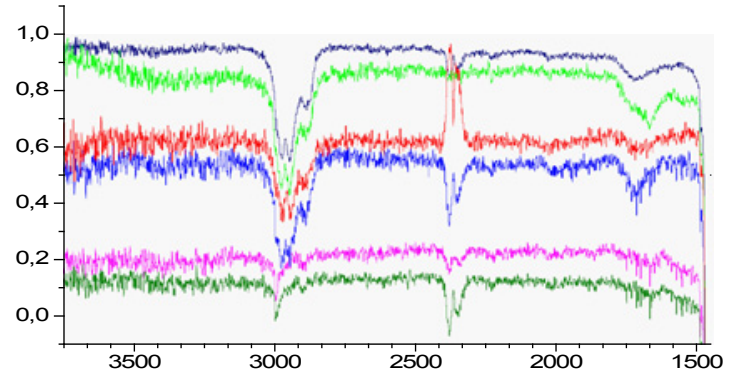

B)

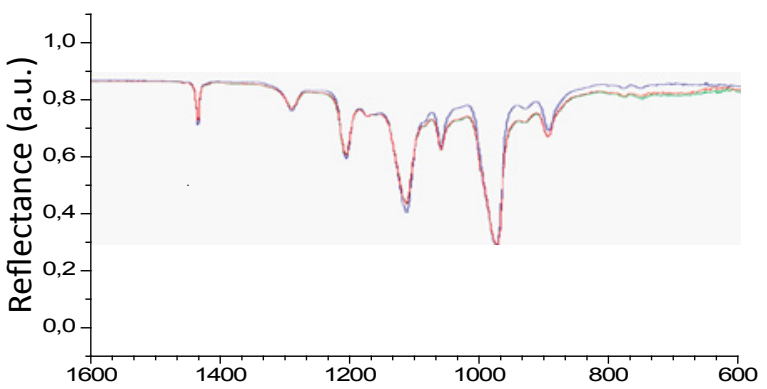

C)

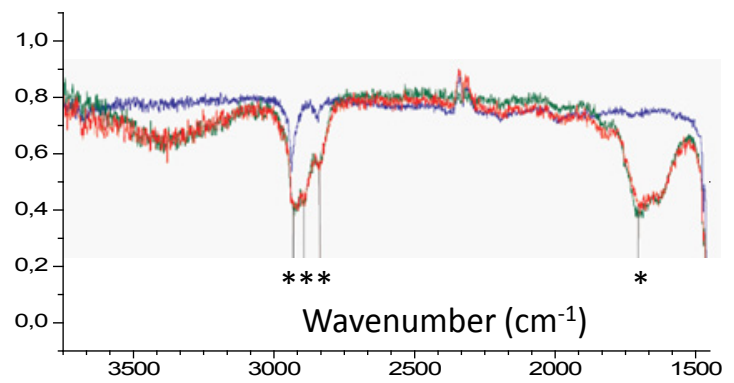

Figure 3: FTIR/ATR spectra of the plasma-treated samples. A): 1-decene treated samples immediately after processing (dark blue), after a single ethanol wash (light green), after a single heptane wash (red) and after three ethanol+heptane washing cycles (light blue). Also shown is reference nonprocessed ETFE (dark green) and reference ETFE after three ethanol+heptane washing cycles (purple). B): HMDSO treated samples immediately after processing (red) and after three ethanol+heptane washing cycles (green), and reference non-processed ETFE (blue). C): n-hexene treated samples immediately after processing (red) and after three ethanol+heptane washing cycles (green), and reference non-processed ETFE (blue).

are presented in Figure 3. For the 1-decene treated sample (Figure 3A) peaks appear in the 3000 to $2800 \mathrm{~cm}^{-1}$ region which are not present in the non-treated ETFE sample shown as dark green (reference) and purple (after 3 ethanol+heptane washing cycles). The dark blue spectrum shows ETFE directly after plasma treatment. The changes are clear, indicating that hydrocarbon groups are on the surface. After washing steps (light green (single ethanol wash), red (single heptane wash) and light blue (three cycles of ethanol+heptane washes) the peaks persist indicating that the chemical structures formed are stable consistent with a covalent modification of the ETFE surface.

The HMDSO treated sample spectra are shown in Figure 3B. The non-treated ETFE (blue) is shown with HMDSO treated (red) and HMDSO treated after three cycles of ethanol+heptane washings). The peaks of the HMDSO interfere with the C-F peaks from ETFE but when low density polyethylene (PE) (Dupont, Geneva, Switzerland) is used as reference instead of ETFE distinct washing cycle resistant peaks at 1230, 1260 and $800 \mathrm{~cm}^{-1}$ appear (results not shown) indicating formation of bonded interactions with the substrate.
Figure $3 \mathrm{C}$ shows the $\mathrm{n}$-hexene treated samples directly after treatment (red) and after three cycles of ethanol+heptane washings (green) together with the untreated reference (blue). After treatment peaks indicated by asterisks emerge at 2970, 2926, and $2870 \mathrm{~cm}^{-1}$ corresponding to $\mathrm{CH}_{3}$ and $\mathrm{CH}_{2}$ groups. The broad peak about $1710 \mathrm{~cm}^{-1}$ corresponds to a partial oxidation of the monomer and perhaps some remaining $\mathrm{C}=\mathrm{C}$ double bonds. Although it is not possible to estimate from FTIR the quantity of the different chemical groups, the spectrum does not change after three washing cycles indicating formation of a stable coating.

The interactions between monomers and the ETFE surface are very complex and simplistic pictures may not adequately describe the interaction. Generally Argon plasma activation will result in defluorination and dehydrogenation of the ETFE and monomers respectively. The resulting free monomer radicals subsequently binds to the ETFE surface as evidenced by FTIR analysis, where spectral features emerging after treatment are resistant to washing procedures indicating formation of stable bonding. Presumably also crossliking between monomers linked to the ETFE also occurs resulting in a bound crosslinked film on the surface.

\section{Partition surface modification affects BLM array maximal lifetime}

We then tested the BLM array average lifetime for membranes formed using partitions modified with the three modification groups. Lipid bilayers were established with the previously described lipid bilayer automation technique (where the bilayer forming solution is applied from the cis side, see Figure 2) [7]. The results are summarized in Figure $4 \mathrm{a}$ ( $n=5-12$ for each type of modification).

All plasma-treated samples were used after three washing cycles. The 'untreated' ETFE partitions were prepainted with the bilayer forming solution (BFS) according to an 'airbrush' procedure where a thin layer of BFS was deposited on the ETFE surface [8]. With prepainted ETFE partitions we obtained maximal lifetimes just above 70 h for $8 \times 8$ BLM arrays. We then investigated single side surface modification mimicking the traditional prepainting with a hydrocarbon solvent. Thus in the assembled bilayer chamber the modified surface always faced the cis compartment. Lifetimes for BLM arrays formed across single-sided HMDSO modified partitions and BLMs formed using 1-decene modified partitions were similar with a maximal lifetime less than $30 \mathrm{~h}$ - significantly lower than for BLM arrays formed across untreated ETFE partitions. For single side $n$-hexene modification maximal membrane array lifetimes were not significantly changed compared to untreated ETFE. However, singlesided $n$-hexene modification resulted in membranes that over time developed higher $\mathrm{C}_{\mathrm{m}}$ values and concomitant low $\mathrm{G}_{\mathrm{m}}$ values compared to all other conditions (see below). This led us to speculate whether we may be able to improve BLM array lifetime induced by $n$-hexene modification by employing modification on both sides of the partitions. Indeed double sided $n$-hexene surface modification greatly improved average membrane array lifetimes, where we obtained a maximal lifetime of 6 days.

\section{Surface modification affects BLM array electrical properties}

We assessed the quality of the BML arrays by characterizing their electrical properties, specifically $\mathrm{G}_{\mathrm{m}}$ and $\mathrm{C}_{\mathrm{m}}$ and the results are presented in Figure $4 \mathrm{~B}$ and $4 \mathrm{C}$. The $\mathrm{G}_{\mathrm{m}}$ and $\mathrm{C}_{\mathrm{m}}$ values for each experimental condition represent the average values as a function of time $\mathrm{T}$ for the fraction of membranes that have survived until T. Membranes formed 
A)

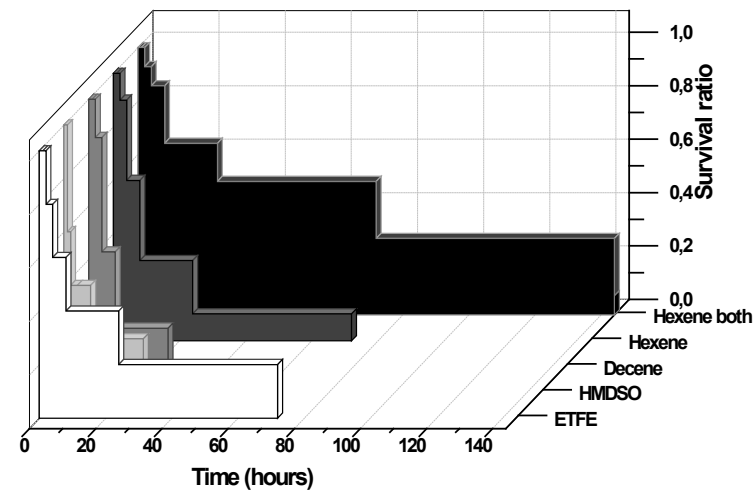

B)

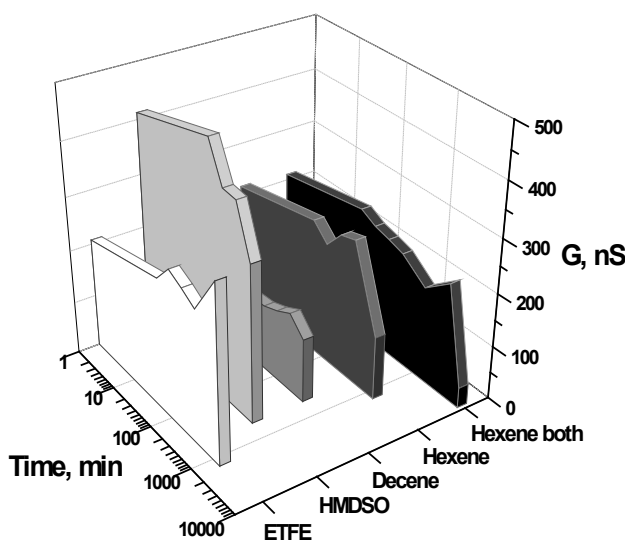

C)

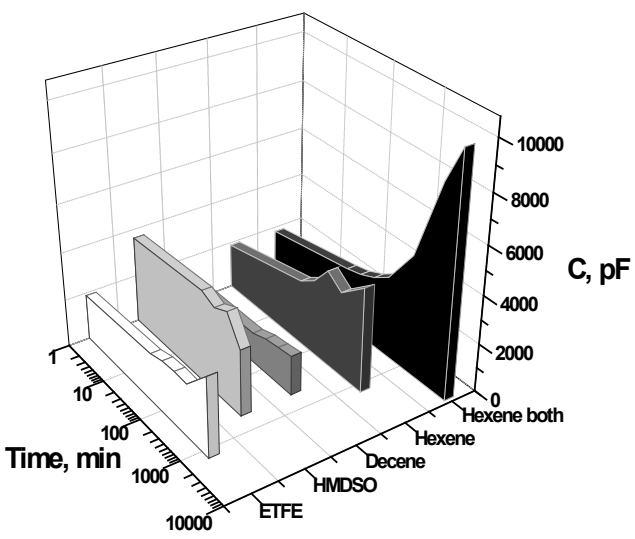

Figure 4: BLM array lifetimes and electrical properties ( $n=58 \times 8$ BLM arrays). A) Survivor plot for BLMs formed across untreated ETFE, single-sided HDMSO-, single-sided 1-decene-, single-sided n-hexene-, and double-sided n-hexene-modified ETFE. The step-wise development reflects the lifetime of each array (i.e. until rupture of the first membrane of the 64 in each array). B-C): Mean $C_{m}$ and $G_{m}$ values for the membranes shown in (A).

in HMDSO coated partitions have significantly higher $G_{m}$ values indicating a higher ionic leak. Lower $G_{m}$ values are seen for membranes formed in the 1-decene coated partitions however this should be seen together with the very low $\mathrm{C}_{\mathrm{m}}$ values which indicated that the effective bilayer membrane area for membranes formed in 1-decene coated partitions is significantly smaller. For the $n$-hexene coated partitions the $G_{m}$ values are comparable to the values found for membranes formed in the untreated ETFE partitions, however over time the $\mathrm{G}_{\mathrm{m}}$ values are reduced significantly indicating that ionic leaks through the membrane is reduced. Comparing $\mathrm{G}_{\mathrm{m}}$ in Figure. $4 \mathrm{~B}$ with $\mathrm{C}_{\mathrm{m}}$ in Figure. $4 \mathrm{C}$ it is clear, that the trend for $\mathrm{G}_{\mathrm{m}}$ follows the trend for $\mathrm{C}_{\mathrm{m}}$ over time for membranes formed in ETFE, HMDSO, and 1-decene coated partitions. This is in contrast to the observations when using $n$-hexene coated partitions. Here a significant increase in $\mathrm{C}_{\mathrm{m}}$ over time is accompanied with a decrease in $G_{m}$. This indicates that for membranes formed in untreated ETFE partitions, HMDSO, and 1-decene coated partitions an increase in membrane area (capacitance) is concomitant with an increase in ionic leak across the membrane. Membranes formed in the $n$-hexene coated partitions show the opposite trend where an increase in membrane area over time is concomitant with a decrease in ionic leak.

Membranes formed in partitions modified on both sides with $n$-hexene evolve from $\mathrm{G}_{\mathrm{m}}=210 \mathrm{nS}$ and $\mathrm{C}_{\mathrm{m}}=1800 \mathrm{pF}$ immediately after membrane formation to $\mathrm{G}_{\mathrm{m}}=40 \mathrm{nS}$ and $\mathrm{C}_{\mathrm{m}}=8640 \mathrm{pF}$ after 140 h. Taking the specific capacitance value of a decane containing lipid bilayer to be $0.4 \mu \mathrm{F} / \mathrm{cm}^{2}$ (Benz et al. 1975), the final conductance of the membrane in double-sided $n$-hexene coated partition corresponds to a bilayer membrane area of $0.02 \mathrm{~cm}^{2}$ which is roughly $50 \%$ of the available aperture area.

In the early phase ( $<24 \mathrm{~h}$ after formation) for double-sided $n$-hexene modified partitions, $\mathrm{C}_{\mathrm{m}}$ is around $1800 \mathrm{pF}$ which could suggest that just a BSF 'glob'is formed. However solvent containing membranes generally undergo a 'thinning' process evidenced as an increase in $\mathrm{C}_{\mathrm{m}}$ over time while $\mathrm{G}_{\mathrm{m}}$ remains constant. We found that double sided modification with $n$-hexene resulted in thinning kinetics in the early phase consistent with the formation of (small) BLM areas in the film, see Figure 5. Thus $n$-hexene wetting of both front and back surfaces is important for BLM array stability - consistent with BLM stabilization originating from the stability of the Plateau-Gibbs border between the BLM and the surrounding hydrocarbon solvent-containing torus [21]. The torus is formed so it contacts both sides of the partition at the rim of each aperture. This is consistent with our observation that only double-sided hexene treatment significantly enhances stability (i.e. slowest release of solvent from the torus) - as none of the single-sided treatments performed here improves stability relative to the case for prepained but otherwise untreated ETFE.

The capacitance increases after about $500 \mathrm{~min}$, but we also note that this increase is over a time period approaching $10.000 \mathrm{~min}$ (cf Figure 2C). Thus the apparent dramatic increase is likely to represent (slow) exclusion of decane from the toruses over time so that the effective bilayer area is increasing.

\section{The stabilizing effect of $\mathbf{n}$-hexene is not related to macroscopic hydrophobicity per se}

$n$-hexene is a hydrophobic molecule and one might expect that surface hydrophobicity per se is responsible for the increased BLM stability and electrical properties. Thus lipid acyl-chains may orient themselves towards the hydrophobic surface creating a lipid mono-layer on the partition surface. In order to obtain insight into the mechanism by which surface modification affected BLM array stability and electrical properties we measured the macroscopic surface hydrophobicity by water contact angle measurements.

None of the hydrophobic monomers applied resulted in an increased hydrophobicity of the ETFE surface. Water contact angles 
Citation: Perry M, Hansen JS, Stibius K, Vissing T, Pszon-Bartosz K, et al. (2011) Surface Modifications of Support Partitions for Stabilizing Biomimetic Membrane Arrays. J Membra Sci Technol S1:001. doi:10.4172/2155-9589.S1-001

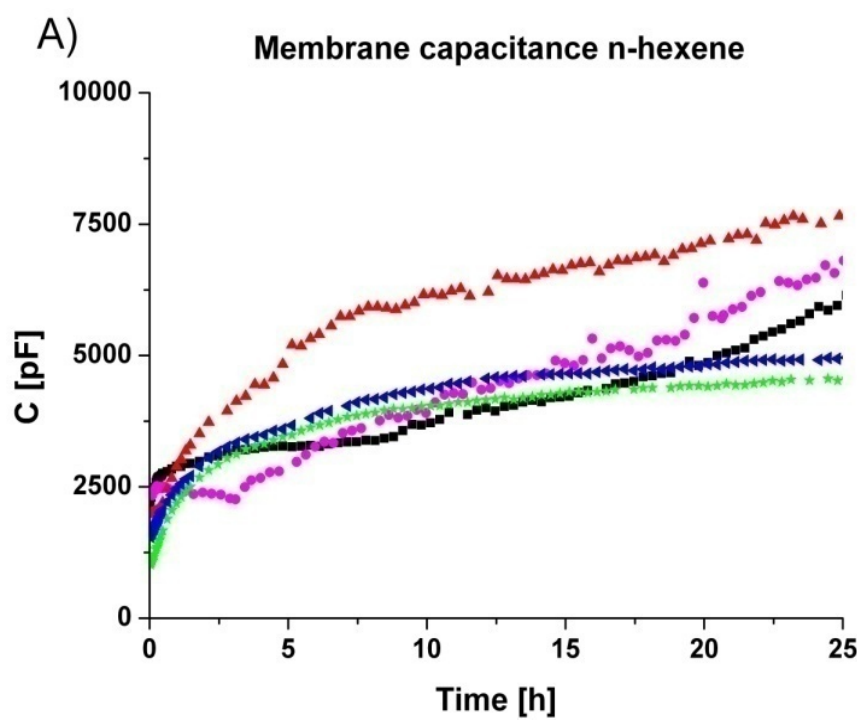

B)

Membrane conductance $n-h e x e n e$

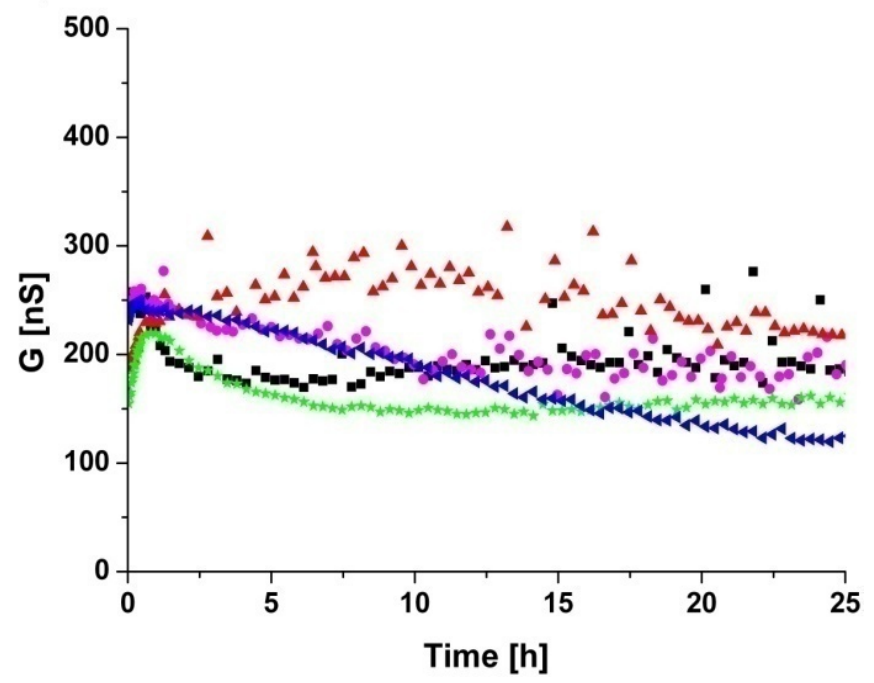

Figure 5: BLM thinning kinetics. A): $C_{m}(t)$ and B): $G_{m}(t)$ values for $n=5$ experiments (shown with 5 different symbols) for $8 \times 8$ BLMs formed across double-sided $n$-hexene modified ETFE partition arrays. Bilayer forming solution: DPhPC in decane, $50 \mathrm{mg} / \mathrm{ml}$.

were reduced by the treatments from $106 \pm 2^{\circ}$ for pure ETFE to $102 \pm 2^{\circ}$ for HMDSO, $98 \pm 2^{\circ}$ for 1 -decene, and $90 \pm 2^{\circ}$ for $n$-hexene. After cleaning with a sequential wash in $60: 40 \mathrm{v} / \mathrm{v}$ EtOH:water, $n$-heptane, and MilliQ water the contact angle values for the modified surfaces decreased slightly to $98 \pm 2^{\circ}$ (HMDSO), $98 \pm 2^{\circ}$ (1-decene), and $89 \pm 2^{\circ}$ ( $n$-hexene) respectively. The large difference in electrical properties $\left(G_{m}\right.$ and $\left.C_{m}\right)$ observed for BLM arrays formed using HMDSO and 1-decene modified partitions is not reflected in the contact angle value which is the same $\left(98^{\circ}\right)$ for both cleaned surfaces. In fact the strongest stabilizing effect was obtained with $n$-hexene which has the lowest contact angle value $\left(89^{\circ}\right)$.

Quantification of $\mathbf{n}$-hexene modification induced changes in partition surface energy

In order to investigate whether the stabilizing effect of $n$-hexene was related to changes in nano-scale surface roughness we characterized the
A)

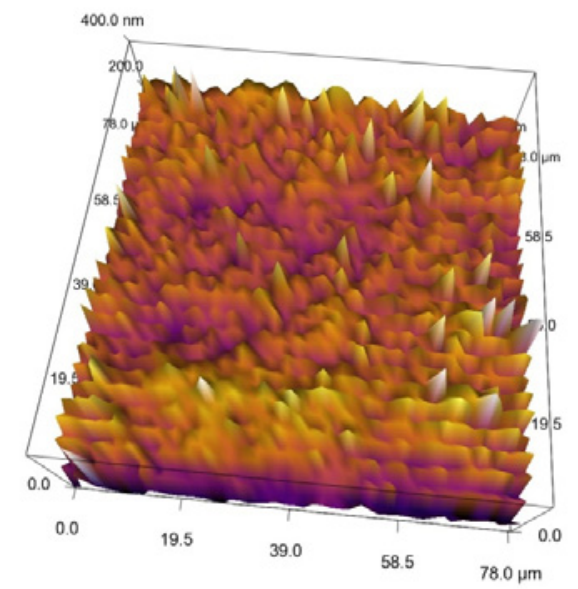

B)

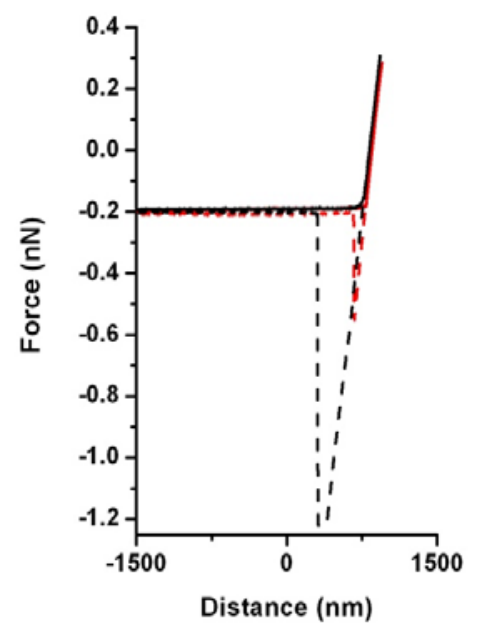

C)

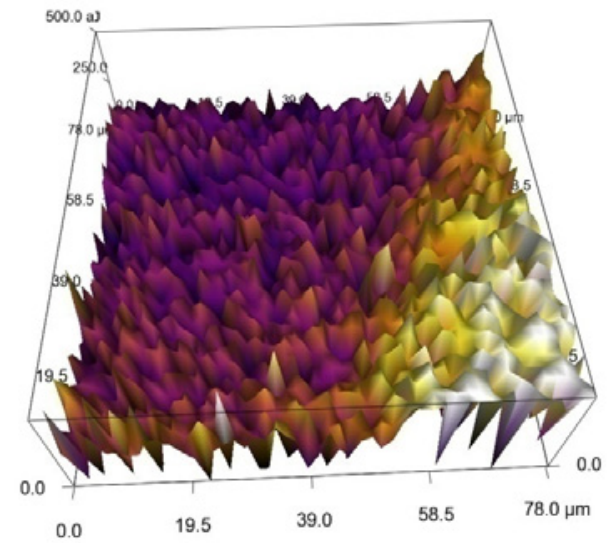

Figure 6: AFM imaging of a $n$-hexene modified partition surface where a triangular area in the lower right corner has been shielded during plasma treatment leaving an unmodified ETFE surface. A): Topographic map. B): Force-distance curves for untreated (black) and $n$-hexene modified (red) partition surface interacting with a hydrophobic (hexadecane coated) AFM tip. Solid lines represent approach while dashed lines represent retraction. C): $50 \times 50$ pixel force volume (FV) map generated from force-distance curves (B) for each pixel for the area shown in $A$. For the untreated surface area the average adhesion energy $W_{a d}=665 \pm 152$ aJ (average of $251 \mathrm{FV}$ measurements), while for the $n$-hexene modified surface $W=345 \pm 90$ aJ (average of $902 \mathrm{FV}$ measurements). For the silicon wafer (natural oxide layer) mounting surface $W_{a d}=122 \pm 21$ aJ (average of $100 \mathrm{FV}$ measurements). 
A)

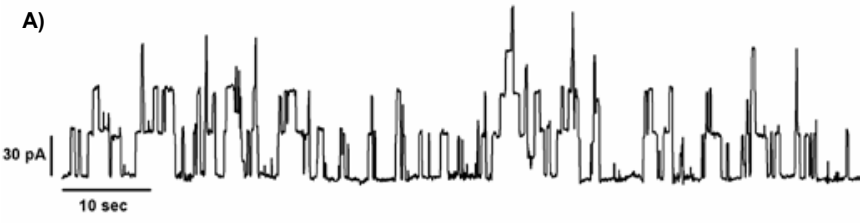

B)

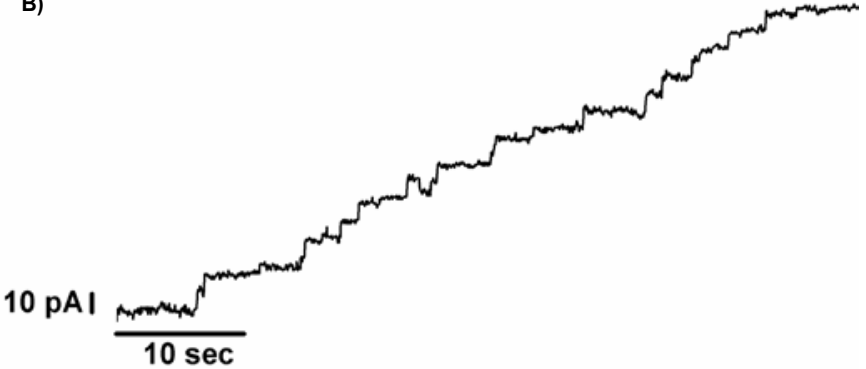

Figure 7: Protein incorporation. A) gA channel current trace in 8x8 BLM array formed across a double-sided n-hexene modified ETFE partition. The BLM array were formed in $1 \mathrm{~N} \mathrm{HCl}$ adjusted to $\mathrm{pH} 1$ using a $50 \mathrm{mg} / \mathrm{ml} \mathrm{DPhPC}$ decane bilayer forming solution with gAadded in a 1:107 ratio. B) $\alpha$-hemolysin channel current trace with experimental details as in (A) except that buffer was replaced with phosphate buffered saline solution (PBS). After BLM array formation $10 \mu \mathrm{l} \alpha$-hemolysin was added to the cis chamber and channel activity recorded as described in the Materials and Methods section.

\begin{tabular}{|l|l|l|}
\hline Surface & Water contact angle Pre wash & Water contact angle Post wash \\
\hline Untreated ETFE & $106^{\circ}$ & $106^{\circ}$ \\
\hline HMDSO & $102^{\circ}$ & $98^{\circ}$ \\
\hline 1-decene & $98^{\circ}$ & $98^{\circ}$ \\
\hline$n$-hexene & $90^{\circ}$ & $89^{\circ}$ \\
\hline
\end{tabular}

Table 1:

surface topography and of non-treated versus $n$-hexene modified ETFE partition surfaces using atomic force microscopy (AFM) topographical measurements. The results are shown in Figure 6A. We found no structural difference between normal and $n$-hexene plasma treated surfaces (see Figure 6A). We then investigated the adhesion energies $W_{a d}$ between a hexadecane functionalized AFM tip (mimicking the lipid molecule acyl chains) and the surfaces by measuring forcedistance relations (see Figure 6B) and obtained a force-volume (FV) map of the ETFE and n-hexene modified surface (see Figure $6 \mathrm{C}$ ). The corresponding $W_{a d}$ values (mean \pm s.d.) were $665 \pm 152$ aJ $(n=251 \mathrm{FV}$ measurements) for the untreated surface and $345 \pm 90$ aJ ( $n=902 \mathrm{FV}$ measurements) for the $n$-hexene treated surface. For comparison for the silicon wafer (natural oxide layer) $W_{a d}=122 \pm 21$ aJ ( $n=100 \mathrm{FV}$ measurements). Thus the decrease in contact angle from $106^{\circ}$ to $89^{\circ}$ corresponds to an almost $50 \%$ decrease in adhesion energy probed with a hexadecane coated surface. The $W_{a d}$ values reflect both the interactions between the interfaces (hexadecane/ $n$-hexene, hexadecane/ETFE, or hexadecane/silicon) and the entropic cost of re-wetting both interfaces upon tip withdrawal.

In general high adhesion energies coincide with high hydrophobicity which is consistent with surface thermodynamics. However the stabilizing effect of $n$-hexene is not due to an increase in hydrophobicity per se; 1-decene modified surfaces which have a higher contact angles in fact results in less stable BLMs. This suggests that the energetics of the interaction between modified surfaces and a lipid/hydrocarbon phase not only relates to macroscopic hydrophobic wetting but also involves contributions due to the amphiphilic nature of the lipids. The stabilizing effect may thus be related to the molecular structure of hexene beyond a mere hydrophobic/ mesoscopic surface topography based mechanism. Since torus stability is related to effective 'sealing' with lipid monolayers interfacing the hydrocarbon containing torus with the aqueous phase, our results suggest that the hexene moieties provides a more favorable interaction with the lipid acyl chains than the other monomers tested.

\section{n-hexene modified ETFE partitions provides BLM arrays with a high current $s / n$ ratio}

To demonstrate that $n$-hexene modified partition aperture arrays combined with the BLM automation technique design are able to support functional incorporation of membrane proteins we reconstituted the peptide gA from B. brevis into a BLM array $24 \mathrm{~h}$ after membrane formation. The pentadecapeptide subunits residing in each monolayer of the lipid bilayer form a cation-selective dimer channel resulting in channel activity as shown in Figure 7A, for a general gA review see [1]. To further demonstrate protein incorporation we established $\alpha$-HL into $8 \times 8$ BLM arrays. The pore of $\alpha-\mathrm{HL}$ is created in the lipid bilayer upon assembly of seven $33 \mathrm{kDa}$ monomeric polypeptides on the membrane surface (Dinges et al. 2000; Fink et al. 1989). Figure 7B shows a current trace after addition of $\alpha-\mathrm{HL}$ demonstrating the formation of $\alpha$-HL pores.

A $\mathrm{G}_{\mathrm{m}}$ value of $40 \mathrm{nS}$ observed for membranes established on double sided $n$-hexene treated partitions corresponds to a resistance of 25 $\mathrm{M} \Omega$ for the entire 64 aperture array. The discrepancy between $25 \mathrm{M} \Omega$ and the giga $\mathrm{Ohm}$ resistance usually recorded for lipid membranes is attributed to the presence of a leak current in our chamber setup. We have previously determined the leak current to be around 50-100nS (data not shown). In other words, when our membrane arrays are completely sealed in all 64 apertures, the current detected by our set-up is the leak current in our system. As soon as a single aperture membrane breaks, ions can freely flow across the partition separating the cis and trans chambers in our set-up and we observe a dramatic increase in the detected current between said chambers. The combination of a sufficiently stable leak current with $G \Omega$ membrane seals still enables us to perform transmembrane ionic current recordings with a high current amplitude signal-to-noise $(s / n)$ ratio.

This suggests that the established BLM arrays may be developed into biosensor arrays where the sensor readout is based on e.g. the activity of single ligand-gated ion channels.

\section{Conclusion}

In this study, we were able to increase the reproducibility and stability of BLM arrays by forming the arrays across ETFE partitions modified using double-sided $n$-hexene plasma polymerization. This demonstrates that the adhesion between the lipid and substrate is an important factor for establishing stable planar lipid bilayer membranes. The bilayer stabilizing adhesion induced by $n$-hexene is not correlated with macroscopic hydrophobicity. The shorter $n$-hexene monomers have a stronger stabilizing effect than the longer more acyl-chain like 1-decene monomer. This suggests that the stabilizing effect is not only determined by hydrophobic wetting, but also by the amphiphilic nature of the lipid molecules. The concomitant low $\mathrm{G}_{\mathrm{m}}$ and high $\mathrm{C}_{\mathrm{m}}$ values for bilayers formed using double-sided $n$-hexene modification enable recordings of transmembrane ionic currents with a high $\mathrm{s} / \mathrm{n}$ ratio. Thus $n$-hexene modification can be used to stabilize large arrays of biomimetic membranes and still enable current recordings with single channel resolution. In conclusion, plasma polymerization 
Citation: Perry M, Hansen JS, Stibius K, Vissing T, Pszon-Bartosz K, et al. (2011) Surface Modifications of Support Partitions for Stabilizing Biomimetic Membrane Arrays. J Membra Sci Technol S1:001. doi:10.4172/2155-9589.S1-001

may be employed to create surface modified scaffolds with improved stability performance for biomimetic membranes. The general versatility in surface modification implies that it can be tailored to meet specific requirements dictated by the choice of biomimetic membrane components.

\section{Acknowledgments}

The work was supported through MEMBAQ, a Specific Targeted Research Project (STREP), by the European Commission under the Sixth Framework Programme (NMP4-CT-2006-033234), by The Danish National Advanced Technology Foundation (023-2007-1) and The Danish National Research Foundation. CHN was also supported by the Environment \& Water Industry Development Council of Singapore (EWI) through project \#MEWR 651/06/169.

\section{References}

1. Andersen OS, Kopppe II RE, Roux B (2007) Gramicidin channels: Versatile tools. In: Chung SH, Andersen OS, Krishnamurthy V (eds) Biological Membrane Ion Channels. Springer, New York, pp 33-80.

2. Behnisch J, Tyczkowski J, Gazicki M, Pela I, Holländer A, et al. (1998) Formation of hydrophobic layers on biologically degradable polymeric foils by plasma polymerization. Surface and Coatings Technology 98: 872-874.

3. Benz R, Fröhlich O, Läuger P, Montal M (1975) Electrical capacity of black lipid films and of lipid bilayers made from monolayers. Biochim. Biophys. Acta 394: 323-334.

4. Dinges MM, Orwin PM, Schlievert PM (2000) Exotoxins of Staphylococcus aureus. Clin Microbiol Rev 13: 16-34.

5. Fink D, Contreras ML, Lelkes PI, Lazarovici $P$ (1989) Staphylococcus aureus alpha-toxin activates phospholipases and induces a Ca2+ influx in PC12 cells. Cell Signal 1: 387-393.

6. Hansen JS, Perry M, Vogel J, Groth J, Vissing T, et al. (2009) Large scale biomimetic membrane arrays. Anal Bioanal Chem 395: 719-727.

7. Hansen JS, Perry M, Vogel J, Vissing T, Ibragimova S, et al. (2009b) Development of Novel Biomimetic Membrane Design. Biophysical Society Meeting Abstracts. Biophysical Journal Supplement 2009: 635a.

8. Hansen JS, Perry M, Vogel J, Vissing T, Geschke O, et al. (2009) Development of an automation technique for the establishment of functional lipid bilayer arrays. J Micromech Microeng 19: 025014

9. Inagaki N, Narushima K, Amano T (2006) Introduction of carboxylic groups on ethylene-co-tetra fluoroethylene (ETFE) film surfaces by CO2 plasma. J Adhes Sci Technol 20: 1443-1462

10. Inagaki N, Narushima K, Kuwabara T, Tamura K (2005) Introduction of amino functionalities on ethylene-co-tetrafluorethylene film surfaces by HN3 plasmas. J Adhes Sci Technol 19: 1189-1205.

11. Inagaki N, Narushima K, Lim SK, Park YW, Ikeda Y (2002) Surface modifications of ethylene-co-tetrafluoroethylene films by remote plasma. J Polym Sci B 40: 2871-2882.

12. Martin DK (2007) Nanobiotechnology of Biomimetic Membranes. Springer Verlag, New York, pp 173.

13. Montal M, Mueller $P$ (1972) Formation of bimolecular membranes from lipid monolayers and a study of their electrical properties. Proc Natl Acad Sci U S A 69: 3561-3566.

14. Mueller P, Rudin DO (1969) Translocators in bimolecular lipid membranes: their role in dissipative and conservative bioenergetic transduction. Curr Topics Bioenergetics 3:157-249.

15. Nielsen CH (2009a) Biomimetic membranes for sensor and separation applications. Anal Bioanal Chem 395: 697-718.

16. Nielsen $\mathrm{CH}$ (2009b) Major Intrinsic Proteins in Biomimetic Membranes. In: Jahn TP, Bienert GP (eds) Major Intrinsic proteins and their role in the exchange of metalloids. Landes Bioscience Publishers Austin TX pp 127-145.

17. Park YW, Inagaki N (2004) A new approach for selective surface modification of fluoropolymers by remote plasmas. J Appl Polym Sci 93: 1012-1020.

18. Perry M, Vissing T, Boesen TP, Hansen JS, Emneus J, Nielsen CH (2009) Automated sampling and data processing derived from biomimetic membranes. Bioinspir Biomim 4: 44001

19. Tien HT, Ottova-Leitmannova A (2003) Planar lipid bilayers (BLMs) and thei applications Membrane Science and Technology Series. Elsevier, Amsterdam, pp 1034.

20. Vogel J, Perry M, Hansen JS, Bolinger PY, Nielsen CH, Geschke O (2009) A support structure for biomimetic applications. Journal of Micromechanics and Microengineering 19: 025026.

21. White SH (1972) Analysis of the torus surrounding planar lipid bilayer membranes. Biophys J 12: 432-445.

22. White SH, Petersen DC, Simon S, Yafuso M (1976) Formation of planar bilayer membranes from lipid monolayers. A critique. Biophys J 16: 481-489.

23. White SH, Thompson TE (1973) Capacitance, area, and thickness variations in thin lipid films. Biochim Biophys Acta 323: 7-22.

24. Yasuda H (1977) Modification of polymers by plasma treatment and by plasma polymerization. Radiat Phys Chem 9: 805-817

25. Yasuda H (1985) Plasma Polymerization. Academic Press, Inc. , Orlando, Fl:

26. Zanini S, Riccardi C, Orlandi M, Esena P, Tontini M, et al. (2005) Surface properties of HDMSO plasma treated polyethylene terephathalate. Surface and Coatings Technology 200: 953-957. 